\title{
Número de séries no treinamento de força muscular: uma breve revisão narrativa de meta-análises sobre adaptações de força e hipertrofia muscular
}

\author{
Leonardo Emmanuel de Medeiros Lima ${ }^{\mathrm{a}}$ (D) , Henrique Miguel ${ }^{\mathrm{bc}}$ (D), Roberto Moriggi Junior ${ }^{\mathrm{def}}$ (iD), \\ Rodrigo Pereira da Silvaghi iD , Henrique Stelzer Nogueirabj iD , José Garcia de Brito-Netok (iD), \\ Dilmar P. Guedes Jrglm iD \\ aGrupo de estudos em treinamento, atividade física e saúde da Universidade São Judas Tadeu, São Paulo, SP, Brasil.

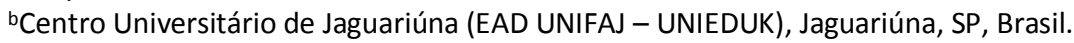 \\ 'Centro Universitário de Espirito Santo do Pinhal (UNIPINHAL), Espirito Sando do Pinhal, SP, Brasil. \\ dFaculdade de Santa barbara D'Oeste (UNIESP), Santa barbara D'Oeste, SP, Brasil. \\ 'Centro Universitário Max Planck (UNIMAX), Indaiatuba, SP Brasil. \\ fFaculdade de Educação Física da UNICAMP (FEF-UNICAMP), Campinas, SP, Brasil. \\ sUniversidade Metropolitana de Santos (UNIMES), Santos, São Paulo, Brasil. \\ hFaculdade Praia Grande (FPG), Praia Grande, São Paulo, Brasil. \\ 'Universidade Federal de São Paulo (UNIFESP), Santos, São Paulo, Brasil. \\ jLaboratório de Biocências da Motricidade Humana - Universidade Federal do Estado do Rio de Janeiro (LABIMH - UNIRIO), Rio \\ de Janeiro, RJ, Brasil. \\ kLiga Acadêmica Multidisciplinar de Oncologia da FACENE (LAMOF), Faculdade Nova Esperança (FACENE/RN), Mossoró, RN, Brasil. \\ 'Universidade Santa Cecilia (UNISANTA), Santo, São Paulo, Brasil. \\ mCentro de Treinamento Fisiologia do Exercício e Treinamento (CEFIT), São Paulo, São Paulo, Brasil.
}

RESUMO Existem recomendações de progressão do treinamento de força muscular, que também considera elevar o número de séries conforme o praticante avança no programa. Este estudo teve por objetivo verificar na literatura, dados de meta-análises que pudessem colaborar com o entendimento sobre a magnitude de ganhos de força e hipertrofia muscular em relação ao número de séries em exercícios de força muscular, de forma tal que este trabalho tem características de breve revisão narrativa. Os resultados foram de que os dados que apontam que a magnitude das adaptações musculares se dá proporcionalmente ao número de séries, e com isso fica entendido de que, apesar de algumas críticas, as recomendações do American College of Sports Medicine são coerentes.

PALAVRAS-CHAVE exercício; treino resistido; treino de força

Aceito 10 de fevereiro de 2021 Publicado online 29 de abril de 2021

Cite este artigo:

Lima et al. (2021) Número de séries no treinamento de força muscular: uma breve revisão narrativa de meta-análises sobre adaptações de força e hipertrofia muscular Multidisciplinary Reviews 4: e2021008. DOI: 10.29327/multi.2021008.

\section{Number of series in muscular strength training: a brief narrative review of meta-analysis on strength adaptations and muscular hypertrophy}

ABSTRACT There are recommendations for the progression of muscle strength training, which also considers increasing the number of sets as the practitioner progresses through the program. This study aimed to verify in the literature, data from meta-analyses that could collaborate with the understanding of the magnitude of strength gains and muscle hypertrophy to the number of sets in muscle strength exercises, in such a way that this work has characteristics brief narrative review. The results were that the data that indicate that the magnitude of muscle adaptations occurs in proportion to the number of series, and with that, it is understood that, despite some criticisms, the recommendations of the American College of Sports Medicine are consistent.

KEYWORDS: exercise, resistance training, strength training 


\section{Introdução}

O treinamento de força (TF) é uma das principais maneiras utilizadas para o ganho da força e hipertrofia muscular (Figueiredo et al 2018). Neste sentido, este praticado regularmente traz uma série de adaptações crônicas relacionadas com a melhora do desempenho esportivo (Lesinski et al 2016; Balsalobre-Fernández et al 2016) e menor risco de mortalidade por diversas causas (Saeidifard et al 2019).

A American College of Sports Medicine (2009) recomenda que indivíduos iniciantes realizem uma a três séries por exercício de oito a 12 repetições com uma carga moderada (70-85\% 1RM), enquanto indivíduos avançados devem realizar três a seis séries por exercício de uma a 12 repetições com uma faixa de carga entre 70-100\% de uma repetição máxima (1RM), com variação de métodos de treino, preferencialmente em uma organização periodizada.

Uma das adaptações morfológicas do músculo esquelético induzido pelo TF é a hipertrofia muscular. A hipertrofia muscular é definida como uma adaptação morfofisiológica, caracterizada por um aumento na área seccional transversa das fibras, decorrente do balanço positivo na razão síntese versus degradação proteica (Nogueira 2018; Lima 2017).

Segundo Figueiredo et al (2018), as adaptações promovidas por meio do TF estão fortemente associadas às variáveis aplicadas dentro do planejamento de sessões de TF. As variáveis que são manipuladas para maximizar a hipertrofia muscular são: intervalo de descanso entre séries, escolha da ordem dos exercícios, número de séries, intensidade de carga, frequência de treinamento e técnicas avançadas. $O$ aumento do volume de treinamento em uma sessão de TF pode ser alcançado por meio do aumento do número de repetições, aumento do número de séries, adição de exercícios, aumento da frequência semanal, ou quando todos estes forem mantidos constantes e a carga total levantada for aumentada.

O volume relativo refere-se ao produto das séries e repetições (expressa por meio do número total de repetições). $O$ volume absoluto (carga total levantada) leva em consideração a intensidade utilizada, referindo-se ao produto das séries, às repetições e à carga utilizada (Zatsiorsky e Kraemer 2008; Zourdos et al 2016). Atualmente já foram publicados diversos estudos e meta-análises referente a manipulação do volume e as respostas da força muscular e hipertrofia, muitos com resultados divergentes entre si. Por tanto, o objetivo deste estudo é verificar na literatura se há uma relação entre o volume no TF, quanto ao número de séries, e o desenvolvimento da força e hipertrofia muscular.

\section{Método}

Este estudo trata-se de uma breve revisão narrativa, com busca diversificada de meta-análises, pelas bases de dados da SciELO, PubMed e Scholar, de forma não sistematizada.

\section{Resultados e Discussão}

Uma das primeiras meta-análises relativa a dose resposta do volume no treinamento de força, foi realizada por Rhea et al (2002), já nesta eles demonstraram que três séries seriam mais eficientes nos ganhos de força na comparação com única série. Posteriormente, o mesmo grupo de pesquisa, Rhea et al (2003), em meta-análise de 140 estudos, apontou que quatro séries promovem maior desenvolvimento de força muscular quando comparados com menos séries.

Novamente, em 2005, os mesmos pesquisadores (Peterson et al 2005) concluíram em sua nova meta-análise que para maiores ganhos de força muscular, indivíduos destreinados deveriam realizar quatro séries, treinados entre quatro e cinco séries, e atletas oito séries para cada grupo muscular, corroborando com os achados de Peterson et al (2004), que identificaram que atletas treinam em média oito séries por grupamento muscular por sessão.

Fischer et al (2013) descrevem que as mesmas adaptações de força e resistência muscular possam ser obtidas por meio da realização de uma única série de oito a 12 repetições quando executadas até falha concêntrica, e que os estudos que concluem que duas e três séries do mesmo exercício promovem resultados superiores quando comparados à apenas uma série, é um fato que ocorre quando não há execução até a falha concêntrica. Em virtude disso, os autores 
criticam as recomendações da ACSM (2009) por entenderem que prescrever múltiplas séries para ganhos de força muscular, ao invés de apenas uma, é algo arbitrário, pois uma meta-regressão de Krieger et al (2009) utilizada como argumentação chave pela publicação da ACSM, é criticada pela inclusão de estudos originais com problemas metodológicos.

Contudo, contradizendo essa informação, Krieger (2010) publicou uma nova meta-análise com estudos mais robustos em que verificou maior tamanho de efeito em $40 \%$ na hipertrofia muscular, quando utilizaram séries múltiplas em comparação com séries únicas. Por fim, reafirmando e complementando as informações anteriores, meta-análise realizada por Schoenfeld et al (2016) encontraram um efeito dose resposta do volume na hipertrofia muscular, recomendando a realização de mais de 10 séries para otimização dos resultados, apontando além disso que para cada série adicionada aumenta-se 0,37\% da massa muscular. Essa meta-análise foi composta por 15 estudos, dos quais 9 eram com indivíduos destreinados, um com sujeitos recreacionalmente treinados, e apenas 5 utilizaram uma amostra treinada em força, dificultando assim a compreensão e estrapolação desses resultados para a população treinada.

Além disso, os estudos anteriores, e estudos incluídos nas meta-análises até então empregavam uma relação de volume abaixo de 10 séries por grupamento muscular. Contudo isso não se reflete no que é encontrado na prática, tendo então pouca validade ecológica. Por exemplo, no estudo de Hackett et al (2013) apresentaram que mais de 95\% dos fisiculturistas entrevistados, por meio de questionário, empregavam aproximadamente 16 séries, divididos entre três a quatro exercícios para cada grupamento muscular, o que representaria algo entre quatro e seis séries para cada exercício, denotando que estes atletas em suas próprias experiências percebem a necessidade de se treinar com mais séries para progredirem no ganho de hipertrofia muscular esquelética. Isso foi relatado também na "Enciclopédia de Fisiculturismo e Musculação" do Arnold Schwarzenegger em colaboração com Bill Dobbins em 1998 (2013), livro esse que inspirou, guiou e ainda guia o treinamento de muitos fisiculturistas.

Atualmente, existem vários estudos na literatura com indivíduos treinados que investigaram mais que 10 séries por grupo muscular por semana (Ostrowski et al 1997; Radaelli et al 2014; Barbalho et al 2019; Schoenfeld et al 2019; Heaselgrave et al 2018; Brigato et al 2019; Aube et al 2020; Scarpelli et al 2020). Entretanto, esses encontraram resultados contraditórios entre si, uns com resultados a favor de mais séries (Radaelli et al 2014; Schoenfeld et al 2019; Brigato et al 2019; Scarpelli et al 2020) outros com resultados apontando que ultrapassar 10 séries semanais por grupo muscular, não proporciona ganhos adicionais (Ostrowski et al 1997; Barbalho et al 2019; Heaselgrave et al 2018; Aube et al 2020).

Por exemplo, no experimento de Schoenfeld et al (2019) utilizaram protocolo de 8-12 repetições realizadas até a falha concêntrica, frequência semanal de 3 dias, realizaram 3, 9 e 15 séries semanais para cada exercício, enquanto, apenas no quadríceps, realizaram 9, 27 ou 45 séries semanais, respectivamente. Todos os grupos apresentaram aumentos nos ganhos de força e resistência sem diferenças entre eles. O aumento da espessura muscular dos extensores do cotovelo foi favorável apenas no grupo 15 séries. 0 volume de até 45 séries semanais por grupamento muscular, também foi favorável nos ganhos hipertróficos, pelo menos no quadríceps, especificamente.

Já para o estudo de Aube et al (2020), foram recrutados 35 homens treinados que realizavam 12, 18 e 24 séries semanais, dentro de um protocolo que durava oito semanas. Ao final do protocolo não foram encontradas diferenças estatisticamente significativas entre os grupos para a resistência de força, massa livre de gordura e espessura do músculo anterior da coxa. Contudo, para a força máxima, o grupo que realizou o protocolo de 18 séries, demonstrou resultados estatisticamente superiores aos outros grupos (12 e 24 séries).

Esses resultados conflitantes de certa forma geram conclusões confusas a esse respeito. Um dos motivos para ser observado um efeito do volume na hipertrofia nas meta-análises e em contra partida, alguns estudos originais não encontrarem diferenças entre grupos de baixo e alto volume, se deve provavelmente ao fato de que esses incluem em suas intervenções uma pequena amostra, levando a falta de poder estatístico, o que tende a torná-los insuficientes para detectar probabilidade estatística quando um efeito pode de fato existir (erro do tipo II) (Schoenfeld e Grgic 2018).

Outra explicação, vem de um estudo recente publicado por Scarpelli et al (2020), neste os voluntários treinados foram divididos em 2 grupos, um que realizou um número de séries relatado frequentemente pela literatura (22 séries) e o outro realizou um aumento de $20 \%$ no número de séries em cima do histórico de treinamento. Nesse estudo foi possível concluir que considerar o histórico de treino para o aumento do volume (aumento de 20\%) foi mais eficaz para 
hipertrofia e ganhos de força do que utilizar um volume relatado pela literatura. Os autores também observaram que no grupo de 22 séries, 4 voluntários reduziram ou não aumentaram o volume de treinamento relativo ao seu histórico de treino (3 reduziram, 1 manteve) o que possivelmente influenciou negativamente nos resultados do grupo. Essa investigação revela a importância da manipulação do volume de treinamento levando em consideração o histórico de treino dos voluntários. $\mathrm{O}$ que nos leva a questionar os estudos publicados anteriormente, principalmente os que não encontraram eficácia do maior volume.

De fato, os dados da literatura nos levam a crer que séries múltiplas são mais eficazes que séries únicas, e que se deve aumentar o volume de treinamento ao longo do processo, entretanto, até que ponto seria viável e sustentável este aumento? Por exemplo, no estudo de Sacarpelli et al (2020) dois voluntários realizavam anteriormente ao estudo, mais de 30 séries para membros inferiores, e um realizava 40 séries. Ao aumentar $20 \%$ acima do seu histórico, 2 desses chegaram a quase 40 séries e 1 a 50 séries para membros inferiores por semana. Algo relativamente complexo de ser realizado e mantido por um longo período, podemos supor que fisiologicamente não é aconselhável manter um volume de treino neste patamar cronicamente.

Por esse mesmo motivo, Schoenfeld e Grgic (2018) sugeriram um modelo teórico de periodização do volume ao longo de um ano. Nesse as 12 primeiras semanas iniciam-se com sete à 10 séries por grupamento muscular por semana. Posteriormente, aumenta-se gradativamente o volume de séries a cada 12 semanas até a 36 semana, chegando a 1520 séries, objetivando evitar o platô do crescimento muscular. Em seguida, é então reduzido o volume de treinamento por duas semanas para duas ou três séries por grupamento muscular, objetivando a manutenção da massa muscular e recuperação das condições físicas. Já foi identificado por Bickel et al (2011) que a redução de 1/9 do volume de séries é suficiente para manutenção da massa muscular. Pois bem, após esse período regenerativo, é então aumentado o número de séries para 20-25 até a $46^{a}$ semana. Por fim, é então reduzido novamente esse volume em $65 \%$, objetivando a supercompensação das proteínas musculares e manutenção muscular (Schoenfeld e Grgic 2018), pois ao que tudo indica diante das evidências, um aumento ininterrupto e sem controle do volume de treinamento não seria capaz de gerar benefícios adicionais devido ao limite anabólico (Dankel et al 2017).

\section{Considerações finais}

A partir dos resultados de meta-análises, é possível concluir que tanto para ganhos de força muscular quanto para hipertrofia, o TF com séries múltiplas é mais interessante do que séries únicas, e que há uma necessidade de considerar o perfil do praticante, pois para iniciantes ou destreinados são recomendadas menos séries, com uma progressão conforme o indivíduo avança para condições de treinado, altamente treinado e atleta.

A manipulação de variáveis como o número de séries, afeta o volume da sessão de treino, que entre outros fatores, deve ser encorajado por meio de uma organização periodizada de forma individualizada, pois além da força e da hipertrofia muscular, deve-se considerar outros elementos fisiológicos inerentes ao TF. Todo esse entendimento está em concordância com as recomendações da ACSM, que apesar de alguma crítica, as demais meta-análises mostram coerência com suas afirmações.

\section{Declaração de conflito de interesse}

Os autores declaram que não há conflitos de interesse.

\section{Financiamento}

Esta pesquisa não recebeu nenhum apoio financeiro.

\section{Referências}

AMERICAN COLLEGE OF SPORTS MEDICINE (2009) Progression Models in Resistance Training for Healthy Adults. Medicine and Science in Sports and Exercise 41:687-708. 
Aube D, Wadhi T, Rauch J, Anand A, Barakat C, Pearson J, Bradshaw J, Zazzo S, Ugrinowitsch C, De Souza EO (2020) Progressive resistance training volume: effects on muscle thickness, mass, and strength adaptations in resistance-trained individuals. Journal of Strength and Conditioning Research - Volume Publish Ahead of Print.

Balsalobre-Fernández C, Santos-Concejero J, Grivas GV (2016) Effects of strength training on running economy in highly trained runners: a systematic review with meta-analysis of controlled trials. Journal of Strength and Conditioning Research 30:2361-2368.

Barbalho MVS, Coswig VS, Steele J, Fisher JP, Paoli A, Gentil P (2019) Evidence for an upper threshold for resistance training volume in trained women. Medicine \& Science in Sports \& Exercise 51:515-522.

Bickel CS, Cross J, Bamman M (2011) Exercise dosing to retain resistance training adaptations in young and older adults. Medicine and Science in Sports and Exercise 43:1177-1187.

Brigatto FA, Lima LEM, Germano MD, Aoki MS, Braz TV, Lopes CR (2019) High resistance-training volume enhances muscle thickness in resistance-trained men. Journal of Strength and Conditioning Research - Volume Publish Ahead of Print.

Dankel SJ, Mattocks KT, Jessee MB, Buckner SL, Mouser JG, Counts BR, Laurentino GC, Loennekeet JP (2017) Frequency: the overlooked resistance training variable for inducing muscle hypertrophy? Sports Med 47:799-805.

Figueiredo VC, Salles BF, Trajano GS (2011) Volume for muscle hypertrophy and health outcomes: the most effective variable in resistance training. Sports Medicine 48:499-505.

Fisher J, Steele J, Bruce-Low S, Smith D (2011) Evidence-based resistance training recommendations. Medicina Sportiva 15:147-162.

Hackett DA, Johnson NA, Chow C (2013) Training practices and ergogenic aids used by male bodybuilders. Journal of Strength and Conditioning Research 27:1609-1617.

Heaselgrave SR, Blacker J, Smeuninx B, McKendry J, Breen L (2019) Dose-response relationship of weekly resistance-training volume and frequency on muscular adaptations in trained men. International Journal of Sports Physiology and Performance 14: 360-368.

Krieger JW (2010) Single vs. multiple sets of resistance exercise for muscle hypertrophy: a meta-analysis. Journal of Strength and Conditioning Research 24:1150-1159.

Krieger JW (2009) Single versus multiple sets of resistance exercise: a meta-regression. Journal of Strength and Conditioning Research 23:1890-1901.

Lesinski M, Prieske O, Granacher U (2016) Effects and dose-response relationships of resistance training on physical performance in youth athletes: a systematic review and meta-analysis. British Journal of Sports Medicine 50:781-795.

Lima WP (2017) Mecanismos moleculares associados à hipertrofia e hipotrofia muscular: relação com a prática do exercício físico. Revista Brasileira de Fisiologia do Exercício 16:95-113.

Mitchell CJ, Churchward-Venne TA, West DWD, Burd NA, Breen L, Baker SK, Phillips SM (2012) Resistance exercise load does not determine training-mediated hypertrophic gains in young men. Journal of Applied Physiology 113:71-77.

Nogueira HS (2018) Conceitos gerais e fatores determinantes para respostas hipertróficas na musculatura esquelética induzidas pelo treinamento de força muscular - uma revisão narrativa. Revista Brasileira de Fisiologia do Exercício 17:60-63.

Ostrowski KJ, Wilson GJ, Weatherby R, Murphy PW, Lyttle AD (1997) The effect of weight training volume on hormonal output and muscular size and function. Journal of Strength and Conditioning Research 11:149-154.

Peterson MD, Rhea MR, Alvar BA (2005) Applications of the dose-response for muscular strength development: a review of metaanalytic efficacy and reliability for designing training prescription. The Journal of Strength and Conditioning Research 19:950-958.

Peterson MD, Rhea MR, Alvar BA (2004) Maximizing strength development in athletes: a meta-analysis to determine the doseresponse relationship. The Journal of Strength and Conditioning Research 18:377-382.

Radaelli R, Botton CE, Wilhelm EN, Bottaro M, Brown LE, Lacerda F, Gaya A, Moraes K, Peruzzolo A, Pinto RS (2014) Time course of low- and high-volume strength training on neuromuscular adaptations and muscle quality in older women. Age (Dordrecht, Netherlands) 36:881-892.

Rhea MR, Alvar B, Burkett L, Ball S (2003) A meta-analysis to determine the dose-response relationship for strength. Medicine and Science in Sports and Exercise 35:456-464.

Rhea MR, Alvar BA, Burkett LN (2002) Single versus multiple sets for strength: a meta-analysis to address the controversy. Research Quartely for Exercise and Sport 73:485-488.

Saeidifard F, Medina-Inojosa JR, West CP, Olson TP, Somers VK, Bonikowske AR, Prokop L, Vinciguerra M, Lopez-Jimenez F (2019) The association of resistance training with mortality: a systematic review and meta-analysis. European Journal of Preventive Cardiology 26:1647-1665. 
Scarpelli MC, Nóbrega SR, Santanielo N, Alvarez IF, Otoboni GB, Ugrinowitsch C, Libardi CA (2020) Muscle hypertrophy response is affected by previous resistance training volume in trained individuals. Journal of Strength and Conditioning Research - Volume Publish Ahead of Print.

Schoenfeld BJ, Contreras B, Krieger J, Grgic J, Delcastillo K, Belliard R, Alto A (2019) Resistance training volume enhances muscle hypertrophy but Not Strength in Trained Men. Medicine and Science in Sports and Exercise 51:94-103.

Schoenfeld BJ, Grgic Z (2018) Evidence-based guidelines for resistance training volume to maximize muscle hypertrophy. Strength and Conditioning Journal 40:107-112.

Schoenfeld BJ, Ogbron D, Krieger JW (2016) Dose-response relationship between weekly resistance training volume and increases in muscle mass: a systematic review and meta-analysis. Journal of Sports Sciences 35:1073-1082.

Schwarzenegger A, Dobbins B (2013) Enciclopédia de fisiculturismo e musculação. 2. ed. Porto Alegre: Artmed.

Zatsiorsky VM, Kraemer WJ (2008) Ciência e prática do treinamento de força. 2. ed. São Paulo: Phorte Editora.

Zourdos MC, Jo E, Khamoui AV, Lee S, Park B, Ormsbee MJ, Panton LB, Contreras RJ, Kim J (2016) Modified daily undulating periodization model produces greater performance than a traditional configuration in powerlifters. Journal of Strength and Conditioning Research 30:784-791. 